\title{
ON LINEAR GROUPS OVER FINITE FIELDS
}

\author{
JI PING ZHANG
}

(Communicated by Warren J. Wong)

\begin{abstract}
Let $G$ be a finite group with an Abelian Sylow $p$-subgroup $P$ $(p>5)$, and $F$, a finite field of characteristic $p$. Set $H=O^{p^{\prime}}(G)$. If $G$ has a faithful $F G$-module $M$ such that $\operatorname{dim}_{F} M<p-2$, then one of the following is true:

(a) $P$ is normal in $G$,

(b) $H / Z(H) \approx \oplus_{i \leq t} L_{2}\left(p^{n_{i}}\right)$, where $n_{i}$ and $t$ are positive integers and $2 t<$ $p-2$,

(c) $p=7$ or 11 and $H \approx 2 . A_{7}$ or $J_{1}$, respectively, $\operatorname{dim}_{F} M \geq p-4$.
\end{abstract}

In 1963, R. Brauer raised forty-three important problems on group and representation theories [2]. The fortieth problem is as follows:

Brauer Problem 40. Determine the linear groups $G$ of small degrees over a finite field $F$.

Let $P$ be a Sylow $p$-subgroup of $G$, where $p$ is the characteristic of $F$. About 25 years ago, Feit began the study of this problem for $|P|=p$ [5], [6]. His results generalized theorems of Brauer [1] and Tuan [4] on ordinary representation. Recently, Blau [11] gave very nice results on the problem when $P$ is cyclic. Since $S L\left(2, p^{n}\right)$ has all $d$ with $2 \leq d \leq p-1$ as the dimension of an irreducible representation over a suitably large finite field of characteristic $p$, it is in general rather difficult to determine the group structure of a linear group over a finite field. In the present paper, under the assumption that $P$ is Abelian, we will characterize the linear groups of degree less than $p-2$ in terms of group theoretical properties. Our results extend the main theorem of Ferguson [10].

All groups in this paper are assumed to be finite, and the notation and terminology are standard and follow that of [7] and [14].

Linear groups of degree at most 4 have been determined [2], [15]. Therefore we will assume in the following that $p$ is greater than 5 .

Lemma 1. Let $G$ be a finite simple group of Lie type. If the characteristic of $G$ is $p$ with $p>5$, and the Sylow p-subgroup of $G$ is Abelian, then $G$ is isomorphic to $L_{2}\left(p^{n}\right)$ forsome integer $n \geq 1$.

Received by the editors September 15, 1989 and, in revised form, December 6, 1989.

1980 Mathematics Subject Classification (1985 Revision). Primary 20C20, 20 E34. 
Proof. It is an easy consequence of the Chevalley commutator identities.

Lemma 2. Suppose $G$ is a finite p-nilpotent group and $V$ is a faithful $F G$ module, where $F$ is a finite field of characteristic $p$. Let $P$ be a Sylow $p$ subgroup of $G$. If $\operatorname{dim}_{F} V<p-1$, then $P$ is normal in $G$.

Proof. If the lemma is not true, let $(G, V)$ be a counterexample such that $|G|+\operatorname{dim}_{F} V$ is minimal. Since $P$ is not normal in $G$, we can choose an element $y \in P \backslash O_{p}(G)$ such that $y^{p} \in O_{p}(G)$ and $Y O_{p}(G)$ is normal in $P$, where $Y=\langle y\rangle$. Set $T=Y O_{p^{\prime}}(G)$. If $T$ is a proper subgroup of $G$, then $\left(T,\left.V\right|_{T}\right)$ satisfies the condition of the lemma. By the minimum of $(G, V), Y$ is normal in $T$. It follows that $Y O_{p}(G)$ is normal in $P O_{p^{\prime}}(G)=G$, contrary to the choice of $y$. So $T=G$. Let $V_{1}, V_{2}, \ldots, V_{s}$ be all composite factors of $V$ and $N_{i}$ be the kernel $\operatorname{Ker} V_{i}$ of $V_{i}$. Then the intersection $\bigcap_{i} N_{i}$ is a subgroup of $O_{p}(G)$. If $s>1$, by the minimum of $(G, V), Y N_{i}$ is normal in $G .\left[y, O_{p^{\prime}}(G)\right] \leq N_{i}$, so $\left[y, O_{p^{\prime}}(G)\right] \leq \bigcap_{i} N_{i} \leq O_{p}(G)$. Therefore the Sylow $p$-subgroup of $G$ is a normal subgroup, contrary to the assumption on $G$. So $V$ is irreducible, $O_{p}(G)=1$, and $P$ is of order $p$. Since $\operatorname{dim}_{F} V<p-1$ and $y-1 \in J(F P)$, the radical of $F P, V(y-1)^{p-2}=0$. By Hall-Higman Theorem $\mathrm{B}, O_{p}(G) \neq 1$, a contradiction. The contradiction proves the lemma.

Theorem 3. Let $G$ be a finite group with an Abelian Sylow p-subgroup $P(p>$ 5) and $F$ an arbitrary finite field of characteristic $p$. Set $H=O^{p^{\prime}}(G)$. If $G$ has a faithful $F G$-module $M$ such that $\operatorname{dim}_{F} M<p-2$, then one of the following must hold:

(a) $P$ is normal in $G$,

(b) $H / Z(H) \approx \oplus_{i \leq t} L_{2}\left(p^{n_{i}}\right)$, where $n_{i}$ and $t$ are positive integers, $2 t<$ $p-2$, and $Z(\bar{H})$ is the center of $H$,

(c) $p=7$ or 11 and $H \approx 2 . A_{7}$ or $J_{1}$, respectively, $\operatorname{dim}_{F} M \geq p-4$.

Proof. Suppose the theorem is not true, and let $G$ be a counterexample of minimal order. Then

1. $P O_{p^{\prime}}(G)$ is $p$-closed; i.e, $P$ is normal in $P O_{p^{\prime}}(G)$.

$P$ is normal in $P O_{p^{\prime}}(G)$ by Lemma 2 .

2. $G=H=O^{p^{\prime}}(G), Z(G)=O_{p^{\prime}}(G) O_{p}(G)$.

Clearly $H=\left\langle P^{x} \mid x \in G\right\rangle$ and by the minimality of $G, H=G$. By (1) $P \leq$ $C_{G}\left(O_{p^{\prime}}(G)\right)$. Hence, $H \leq C_{G}\left(O_{p^{\prime}}(G)\right)$. Now $H=G$ yields $O_{p^{\prime}}(G) \leq Z(G)$. Similarly, since $P$ is Abelian, $O_{p}(G) \leq Z(G)$.

3. $G=F^{*}(G)$, the generalized Fitting subgroup of $G$, and $G$ is perfect; i.e, $G^{\prime}=G$.

By (2) and the definition of $F^{*}(G), \overline{F^{*}(G)}=F^{*}(G) / Z(G)=\bar{N}_{1} \times \bar{N}_{2} \times$ $\cdots \times \bar{N}_{s}$, where $Z(G) \leq N_{i}$ and $\bar{N}_{i}$ is non-Abelian simple and contains $p$ as a prime divisor of its order. Let $y$ be an arbitrary element of $P$. $P \cap$ $F^{*}(G)=P_{1} P_{2} \cdots P_{s}$, where $P_{i}$ is a Sylow $p$-subgroup of $N_{i}$. Since $P$ is Abelian, $\left[y, P_{i}\right]=1$. For each $N_{i}, \bar{N}_{i}^{y}$ is also normal in $\overline{F^{*}(G)}$ and $P_{i}$ is 
contained in $N_{i} \cap N_{i}^{y}$, so $\bar{N}_{i}^{y}=\bar{N}_{i}$. By [16], $y$ induces an inner automorphism of $\bar{N}_{i}$, so there exists a $p$-element $x_{i}$ of $N_{i}$ such that $\overline{y x_{i}}$ centralizes $\bar{N}_{i}$. Then $y x_{i}$ centralizes $N_{i}$. It follows that $\left.y\left(x_{i} x_{2} \cdots x_{s}\right) \in C_{G} F^{*}(G)\right) \leq F^{*}(G)$, $y \in F^{*}(G)$. By $(2), F^{*}(G)=G$. Now it is easy to see that $G$ is perfect by the minimum of $G$.

4. $G / Z(G)$ is non-Abelian simple.

If $G / Z(G)$ is not simple, then $G / Z(G) \approx \bar{M}_{1} \times \bar{M}_{2} \times \cdots \times \bar{M}_{t}, t \geq 2, M_{i}$ contains $Z(G)$ as a subgroup, and $\bar{M}_{i}$ is non-Abelian simple. By the minimum of $G$, the theorem is true for $M_{i}$. If there is $i$, say $i=1$, such that $M_{i}^{\prime} \approx 2 . A_{7}$ for $p=7$ then, by Blau [11], $\operatorname{dim}_{F} M \geq 4$. Hence $M_{2}^{\prime}$ is isomorphic to either 2. $A_{7}$ or $L_{2}\left(7^{n}\right)$. If $M_{2}^{\prime}$ is isomorphic to $2 . A_{7}$, then $\left(M_{1} M_{2}\right)^{\prime}$ is a homomorphism image of $2 . A_{7} \times 2 . A_{7}$. Every nontrivial $F\left(2 . A_{7} \times 2 . A_{7}\right)$-module $U$ is of dimension at least $4+4=8$. It follows that $7-3=4 \geq \operatorname{dim}_{F} M \geq$ $4+4=8$, which is absurd. If $\bar{M}_{2}$ is isomorphic to $L_{2}\left(7^{n}\right)$, then that will lead to a similar contradiction on dimensions. Similarly, there exists no $i$ such that $M_{i}^{\prime} \approx J_{1}$ with $p=11$. Therefore $M_{i} / Z(G)$ is isomorphic to $L_{2}\left(p^{n_{i}}\right)$ for some positive integer $n_{i}$. Since $\operatorname{dim}_{F}\left(\left.M\right|_{M_{i}}\right) \geq 2,2 t<p-2$. This shows that the theorem is true for $G$. This contradicts the assumption on $G$.

5. $P$ is not cyclic.

This follows obviously from (4) and [11].

6. Last contradiction.

Set $\bar{G}=G / Z(G)$. If $\bar{G}$ is isomorphic to $A_{n}(n \geq 5)$, then by (5) $2 p \leq$ $n \leq p^{2}-1$. There is a subgroup $B_{0}$ of $G$ such that $Z(G) \leq B_{0}$ and $\bar{B}_{0}$ is isomorphic to $A_{p} \times A_{p}$. Notice that $p>5,\left.\operatorname{dim}_{F} M\right|_{B_{0}} \geq 2(p-3) . p-3 \geq$ $2(p-3)$. This, too, is absurd.

If $\bar{G}$ is isomorphic to $G(q)$, a simple group of Lie type, and $q$ is a power of a prime $r$, then by Lemma $1 p$ is not equal to $r$. If $\bar{G}$ is isomorphic to $\operatorname{PSL}(n, q)$, then by [12], with $p>5, p-3 \geq(q-1) / d$ for $n=2$ or $p-3 \geq q^{n-1}-1$ for $n>2$, where $d=(2, q-1)$. If $n=2, p \geq$ $(q-1) / d+3=(q+3 d-1) / d$. So $p>(q-1) / d$. Since $p$ is a prime divisor of $|\bar{G}|, p \mid(q+1) / d$. Hence $(q+3 d-1) / d \leq p \leq(q+1) / d$, which is absurd. If $n>2$, then $p \geq q^{n-1}+2$. Since $p \mid\left(q^{i}-1\right)$ for some positive integer $i \leq n, p \mid\left(q^{n}-1\right) /(q-1)$. Suppose $\left(q^{n}-1\right) /(q-1)=t p$. If $t \geq 2$, then $q \leq t(q-1) \cdot q^{n}-1=t p(q-1) \geq q\left(q^{n-1}+2\right)$, which is absurd. So $t=1$, $p=\left(q^{n}-1\right) /(q-1)$. Then $P$ is of order $p$, contrary to (5). Similarly, $G$ is not isomorphic to any one of the following groups: $\operatorname{PSP}(2 n, q), P S U(n, q)$, $\operatorname{PSO}^{+}(2 n, q)^{\prime}, \operatorname{PSO}^{-}(2 n, q)^{\prime}, P S O(2 n+1, q), G_{2}(q), E_{6}(q), E_{7}(q), E_{8}(q)$, $F_{4}(q)$.

If $\bar{G}$ is isomorphic to ${ }^{2} F_{4}(2)^{\prime}$, then $p=13$. Hence $P$ is of order 13, contrary to (5). If $\bar{G}$ is isomorphic to ${ }^{2} F_{4}(q), q=2^{2 m+1}, m \geq 1$, then by [12] $p-3 \geq(q / 2)^{1 / 2} q^{4}(q-1), p \geq q^{4}+1$. The order of ${ }^{2} F_{4}(q)$ is $q^{12}\left(q^{6}+1\right)\left(q^{4}-1\right)\left(q^{3}+1\right)(q-1)$, so $p \mid\left(q^{6}+1\right)$. Since $q^{6}+1=\left(q^{2}+1\right)\left(q^{4}-q^{2}+1\right)$, 
$p \leq q^{4}-q^{2}+1$, contradicting $p \geq q^{4}+1$. By a similar argument, we can show that $\bar{G}$ is not isomorphic to any one of the following groups: ${ }^{2} E_{6}(q),{ }^{3} D_{4}(q)$, $S z(q),{ }^{2} G_{2}(q)$.

By the classification of finite simple groups, $\bar{G}$ is isomorphic to a sporadic simple group. It is easy to check by the Atlas [14] that $\bar{G}$ is isomorphic to $\mathrm{Co}_{1}$, $B$, or $T h$ with $|P|=49$ or $F_{1}$ with $|P|=121$. There exists an extra-special 2-subgroup of order $2^{1+8}$ in each of the four simple groups. It follows that $\operatorname{dim}_{F} M \geq 2^{4}$. So $11-3 \geq p-3 \geq 16$, which is absurd. The contradiction proves the theorem.

Corollary 4. Suppose $G$ is a finite group with an Abelian Sylow p-subgroup $P(p>11)$. If $G$ has a faithful $F G$-module of degree at most $p-3$ over a field $F$ of characteristic $p$, then either $P$ is normal in $G$ or $O^{p^{\prime}}(G) / Z\left(O^{p^{\prime}}(G)\right)$ is isomorphic to $\oplus_{i \leq t} L_{2}\left(p^{n_{i}}\right), n_{i} \geq 1,2 t<p-2$.

This result is similar to that of Ferguson [10].

Remark. If $F$ is of characteristic zero, that $P$ is Abelian directly follows that $\operatorname{dim}_{F} M<p-2$. But in the modular case, if we did not assume that $P$ is Abelian, there would be many simple groups added to the list, which would make Theorem 3 less meaningful.

\section{ACKNOWLEDGMENTS}

This paper was written during the author's visit of the author to the University of Florida. The author would like to thank the Department of Mathematics of the University for its hospitality. The author also thanks the referee for many useful comments.

\section{REFERENCES}

1. R. Brauer, On groups whose orders contain a prime to the first power, I, II, Amer. J. Math. 64 (1942), 401-440.

2. __ Representations of finite groups, Lectures on Modern Mathematics I, Wiley, 1963.

3. R. Brauer and H. F. Tuan, On simple groups of finite order, Bull. Amer. Math. Soc. 51 (1945), 756-766.

4. H. F. Tuan, On groups whose orders contain a prime to the first power, Ann. of Math. $\mathbf{4 5}$ (1944), 110-140.

5. W. Feit, Groups with a cyclic Sylow p-subgroup, Nagoya Math. J. 27 (1966), 57!-584.

6. __ On finite linear groups, J. Algebra 5 (1967), 378-400.

7. _ The representation theory of finite groups, North-Holland, Amsterdam, 1982.

8. J. P. Zhang, Complex linear groups of degree at most $p-1$, Contemp. Math., vol. 82, 1989, pp. 243-254.

9. __ On linear groups of degrees at most $|P|-1, \mathrm{~J}$. Algebra (to appear).

10. P. Ferguson, Complex linear groups of degree at most $v-3$, J. Algebra 92 (1985), 246-252.

11. H. I. Blau, Minimal modular character degrees for groups with a cyclic Sylow p-subgroup (to appear). 
12. V. Landazuri and G. M. Seitz, On the minimal degrees of projective representations of the finite Chevalley groups, J. Algebra 32 (1974), 418-443.

13. R. W. Carter, Simple groups of Lie type, Wiley-Interscience, New York, 1972.

14. J. H. Conway, R. T. Curtis, S. P. Norton, R. A. Parker, and R. A. Wilson, Atlas of finite groups, Clarendon Press, Oxford, 1985.

15. H. F. Blichfeldt, Finite collineation groups, Univ. of Chicago Press, Chicago, 1917.

16. F. Gross, Automorphisms which centralize a Sylow p-subgroup, J. Algebra 77 (1982), 202233.

Department of Mathematics, Peking University, Beijing, China 